\title{
Nonexistence of a Class of Variate Generation Schemes
}

\author{
Shane G. Henderson, Cornell University \\ Peter W. Glynn, Stanford University
}

November 14, 2001

\begin{abstract}
Motivated by a problem arising in the regenerative analysis of discrete-event system simulation, we ask whether a certain class of random variate generation schemes exists or not. Under very reasonable conditions, we prove that such variate generation schemes do not exist. The implications of this result for regenerative steady-state simulation of discrete-event systems are discussed.
\end{abstract}

\section{Introduction}

Consider a sequence of independent $\operatorname{Bernoulli}(p)$ random variables $X_{1}, X_{2}, \ldots$, so that $P\left(X_{i}=1\right)=p=1-P\left(X_{i}=0\right)$ for all $i \geq 1$. Suppose that $p \in[0,1 / 2)$ but that you have no further information about $p$. Can you then generate a Bernoulli $(2 p)$ random variable in finite time?

This question arises in relation to the initial transient problem for the steadystate simulation of regenerative processes $[2,11]$. It is also a distillation of a problem arising in relation to identifying regeneration times in the regenerative steady-state simulation of discrete-event systems.

For many systems, regeneration times are easily determined by observing the sample paths. For example, in a Markov chain, successive visits to a fixed state constitute regeneration times and can be used in steady-state simulation analysis so long as the state is visited infinitely often. However, in general it is difficult to identify regeneration times. Consider, for example, a queue with an arrival process that is the superposition of several renewal arrival processes with nonexponential continuous interarrival time distributions. It is not clear that such a system can exhibit any regenerative structure.

Nevertheless, regeneration times are known to exist for any "well-posed" simulation [6], including the superposition process alluded to above under moderate conditions; see $[19,5]$. The difficulty is that they may be hard to identify. This problem is discussed in detail in [10] in the context of discrete-event system simulation.

The identification of such regeneration times is based on modeling a discreteevent system as a general state space Markov chain (GSSMC). The GSSMC is obtained by observing the discrete-event system at event times, and incorporating enough state information so that the resulting process is Markov. One then uses a "splitting" technique introduced in $[16,3]$ (see also $[1,12]$ ) to identify the regenerations. All of the known methods for employing the splitting technique require 
explicit knowledge of the $m$-step transition kernel of the GSSMC for some $m \geq 1$. When $m>1$, this transition kernel is unlikely to be easily computed except in very special situations, but the $m>1$ case is perhaps the most likely situation in practice [10].

So one might reasonably ask whether these regeneration times can be computed without explicit knowledge of the m-step transition kernel. This is the question that we address in this paper. As we will show, the answer is, in great generality, no. One needs explicit knowledge of the $m$-step transition kernel to be able to identify these regenerations.

Section 2 explains in greater detail how this problem arises within the regenerative simulation setting, and can be skipped if the reader prefers. Section 3 places the problem in a slightly more abstract setting and provides two nonexistence proofs under different assumptions about one's level of knowledge about the transition kernel. Section 4 describes the implications of these results for the regenerative method of steady-state simulation. It also revisits the Bernoulli example given above to answer the question posed there, partly with the help of the elegant results of [11].

\section{Regenerative Simulation of Discrete-Event Systems}

A generalized semi-Markov process (GSMP) is a stochastic process evolving on a countable state space, and may be used to model a wide variety of discrete-event systems. We give only a very brief description of GSMPs here. More detailed descriptions may be found in, for example, $[17,8,10]$.

The GSMP has piecewise constant sample paths, and the time between jumps of the sample paths are determined by a set of active events associated with each system state. While in a given state, clocks associated with the active events decrease at unit rate until one or more of them reach 0 . When the first clock reaches 0 , the GSMP jumps to a new state, and some clock readings may be added or discarded.

A GSMP may be formally defined and analyzed through a related GSSMC. The GSSMC records both the GSMP state and the vector of active clock readings at the time of state transitions of the GSMP. Under very mild conditions [6] the GSSMC is known to be positive Harris recurrent, and [8] provides easily verifiable conditions for positive Harris recurrence in the case where the state space of the GSMP is finite.

Definition 1 Let $X=\left\{X_{n}: n \geq 0\right\}$ be a Markov chain on a complete separable metric space $\Sigma$. We say that $X$ is Harris recurrent if there exists an $m \geq 1$, a set $A \subseteq \Sigma, a \lambda>0$ and a probability distribution $\varphi$ such that:

$$
\begin{aligned}
& \text { 1. } P^{m}(x, \cdot) \triangleq P\left(X_{m} \in \cdot \mid X_{0}=x\right) \geq \lambda \varphi(\cdot) \quad \forall x \in A \text {; and } \\
& \text { 2. } P\left(X_{n} \in A \text { infinitely often } \mid X_{0}=x\right)=1 \quad \forall x \in \Sigma \text {. }
\end{aligned}
$$

Remark 1 It is often possible to explicitly identify $A, \lambda$ and $\varphi$ that satisfy the above requirements.

Harris chains automatically possess a unique (up to a multiplicative constant) nontrivial $\sigma$-finite invariant measure $\pi$. 
Definition 2 Suppose that $X$ is Harris recurrent with invariant measure $\pi$. If $\pi$ has finite total mass then we say that $X$ is positive Harris recurrent, and we may take $\pi$ to have total mass 1 , so that it is a probability.

We give a brief overview of how regeneration times for a Harris recurrent chain $X$ may be constructed using splitting. For a more careful account, see [7, 12]. For $x \in A$, we may write

$$
P^{m}(x, \cdot)=\lambda \varphi(\cdot)+(1-\lambda) Q(x, \cdot),
$$

where $Q(x, \cdot)$ is given by

$$
\frac{P^{m}(x, \cdot)-\lambda \varphi(\cdot)}{1-\lambda}
$$

if $\lambda<1$, and (arbitrarily) a point mass at $x$ otherwise.

This decomposition suggests that starting from $X_{0}=x \in A$, we could generate $Z_{0}$ as a $\operatorname{Bernoulli}(\lambda)$ r.v. If $Z_{0}=1$, then $X_{m}$ should be generated from $\varphi$, but otherwise $X_{m}$ should be generated from $Q(x, \cdot)$. If $Z_{0}=1$, then $X_{m}$ will be distributed according to $\varphi$ independently of $X_{0}$, so that in fact, $\left(X_{m}, X_{m+1}, \ldots\right)$ and $X_{0}$ are independent, and $m$ is (in a certain sense) a regeneration time. Notice however, that after generating $X_{0}$ and $X_{m}$, we must generate $X_{1}, \ldots, X_{m-1}$ conditional on those two values, which may be difficult. Fortunately, an alternative approach is available.

First, generate $X_{0}, \ldots, X_{m}$. Then compute a Bernoulli r.v. $Z_{0}$ with success probability $w\left(X_{0}, X_{m}\right)$, where $w(x, \cdot)$ is a density of $\lambda(x) \varphi(\cdot)$ with respect to $P^{m}(x, \cdot)$. If $Z_{0}=1$, then $X_{m}$ is distributed according to $\varphi$ independently of $X_{0}$, and a regeneration is recorded. If not, then $X_{m}$ is distributed according to $Q(x, \cdot)$.

Either of these methods may be applied to determine regeneration times for $X$. Notice that both methods require the ability to generate random variates from $Q(x, \cdot)$ for $x \in A$. In the first method this requirement is explicit, while in the second it is implicit. If one can implement the second method, then one can repeatedly generate $X_{1}, \ldots, X_{m}$ conditional on $X_{0}=x$, and compute the Bernoulli r.v. $Z_{0}$ until $Z_{0}=0$ in standard acceptance/rejection fashion.

So then it appears that we can define regeneration times for the GSSMC, but there is a problem. Both methods for determining regeneration times require information on $P^{m}(x, \cdot)$ for $x \in A$. When $m=1$, this presents little difficulty, since $P^{1}(x, \cdot)$ is easily computed. But $P^{m}(x, \cdot)$ is typically extremely difficult to compute when $m>1$. Unfortunately, it is shown in [10] that we can expect the $m>1$ case to be the norm rather than the exception in practice.

So we see that to apply the regenerative method using either one of the two methods outlined above, we need the ability, either directly or indirectly, to generate random variates from $Q(x, \cdot)$. We may assume that we can compute $\lambda$ and $\varphi$, and that we have the ability to generate random variates from $P^{m}(x, \cdot)$, but that we do not have the ability to (exactly) compute $P^{m}(x, \cdot)$. So can we generate random variates from $Q(x, \cdot)$ under these circumstances?

\section{Two Nonexistence Results}

To attack this problem we first generalize the setting. Suppose that $P=\lambda \varphi+(1-$

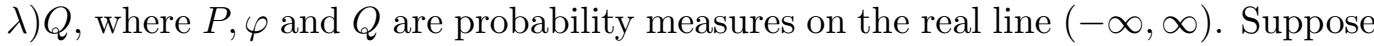
that $\lambda \varphi$ can be computed and $P$ cannot, but independent random variates from $P(\cdot)$ can be generated. The goal is to generate a random variate from $Q(\cdot)$. 
Remark 2 In order for this problem to make sense, the assumed knowledge must uniquely determine the probability measure $Q$. If one can generate independent variates from $P(\cdot)$, then one can estimate the distribution function $F$ of $P$ by the empirical distribution function $F_{n}$ of $n$ independent variates from $P$. As $n \rightarrow \infty$, the Glivenko-Cantelli theorem (see Theorem 20.6 on page 275 of [4] for example) asserts that

$$
\sup _{x \in \mathbb{R}}\left|F_{n}(x)-F(x)\right| \rightarrow 0
$$

almost surely. Thus the distribution function $F$ is completely determined in the limit, and therefore so is $P$. Furthermore, if $\lambda \varphi(\cdot)$ is known, then $\lambda=\int_{\mathbb{R}} \lambda \varphi(d x)$ is also known. Thus $Q$ is uniquely determined as long as we avoid the trivial case where $\lambda=1$, which we henceforth assume.

Suppose that the measure $\lambda \varphi(\cdot)$ on $(\mathbb{R}, \mathcal{B})$ is fixed and given, where $\mathcal{B}$ denotes the usual Borel sigma field. Let $\mathcal{P}_{0}$ be the class of all probability measures on $(\mathbb{R}, \mathcal{B})$ such that $P(\cdot) \geq \lambda \varphi(\cdot)$. We desire an algorithm that works for all $P \in \mathcal{P}_{0}$. The algorithm does not know what $P$ is, but can take advantage of variates generated from $P$, plus other independent random variables.

So, let us construct a probability space as follows. Let $\Omega=\bigotimes_{i=1}^{\infty}(\mathbb{R} \times[0,1])$ denote the sample space, and equip $\Omega$ with the usual product sigma field. Each $\omega \in \Omega$ takes the form

$$
\omega=\left(\left(x_{1}, u_{1}\right),\left(x_{2}, u_{2}\right), \ldots\right) .
$$

For $i \geq 1$, define the random variables $X_{i}(\omega)=x_{i}$ and $U_{i}(\omega)=u_{i}$. For $P \in \mathcal{P}_{0}$, let

$$
P_{P}\left(\left(d x_{1}, d u_{1}\right), \ldots,\left(d x_{n}, d u_{n}\right)\right)=\prod_{i=1}^{n} P\left(d x_{i}\right) I\left(0 \leq u_{i} \leq 1\right) d u_{i} .
$$

Hence, under $P_{P}, X=\left(X_{i}: i \geq 1\right)$ is an i.i.d. sequence of random variables distributed according to $P, U=\left(U_{i}: i \geq 1\right)$ is an i.i.d. sequence of uniform $(0,1)$ random variables, and $X$ and $U$ are independent. The $U$ sequence permits us to randomize the algorithm.

Let $T$ be a finite-valued stopping time adapted to the filtration $\left(\mathcal{F}_{n}: n \geq 1\right)$, where, for $n \geq 1, \mathcal{F}_{n}=\sigma\left(\left(X_{i}, U_{i}\right): 1 \leq i \leq n\right)$. Here $T$ is the computational timehorizon or termination time for the algorithm. Note that we want to incorporate time-horizons that are potentially random, but $T$ should not depend on the entire sequence $\left(\left(X_{i}, U_{i}\right): i \geq 1\right)$. Let $Z$ be $\mathcal{F}_{T}$ measurable, so that $Z$ can be computed solely from the sample path up to time $T$. Our goal is to find a pair $(Z, T)$ with the property that for all $P \in \operatorname{cal} P_{0}$,

$$
P_{P}(Z \in \cdot)=\frac{P(\cdot)-\lambda \varphi(\cdot)}{1-\lambda}
$$

We begin by answering the same question for $P \in \mathcal{P}_{1}$, where $\mathcal{P}_{1}$ is a strict subset of $\mathcal{P}_{0}$. This corresponds to demanding more information about $P$ than just that it lie in $\mathcal{P}_{0}$. Suppose that $\varphi$ has a density $\phi$ (with respect to Lebesgue measure). Define $\mathcal{P}_{1}$ to be the subset of $\mathcal{P}_{0}$ in which $P$ has a density $p$ say, so that a typical $P \in \mathcal{P}_{1}$ has a density of the form

$$
p=\lambda \phi+(1-\lambda) q,
$$

where $q$ is also a density function. 
Theorem 1 Suppose that $\lambda \in(0,1)$ and $\varphi$ has a density $\phi$. Then there does not exist a pair $(Z, T)$ as defined above that satisfies property (3) for all $P \in \mathcal{P}_{1}$.

Proof: Let $P_{1}, P_{2} \in \mathcal{P}_{1}$ with densities $p_{1}$ and $p_{2}$ so that

$$
\begin{aligned}
& p_{1}=\lambda \phi+(1-\lambda) q_{1}, \text { and } \\
& p_{2}=\lambda \phi+(1-\lambda) q_{2},
\end{aligned}
$$

where $q_{1}$ and $q_{2}$ are densities of probability measures $Q_{1}$ and $Q_{2}$ respectively.

We further select $P_{1}$ and $P_{2}$ so that for $i=1,2, q_{i}>0$ only when $\phi>0$, and so that the supports of $q_{1}$ and $q_{2}$ are disjoint; see Figure 1.

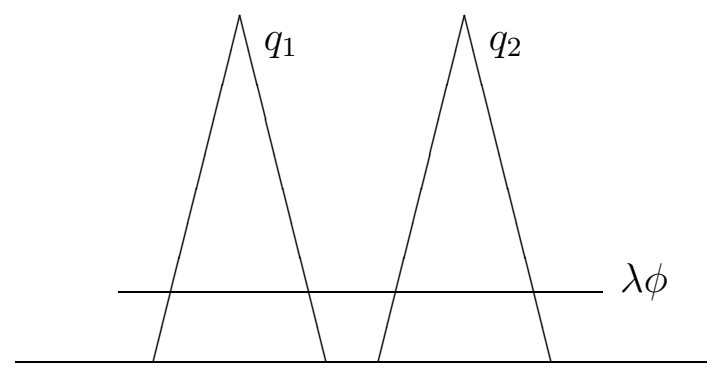

Figure 1: The densities $\lambda \phi, q_{1}$ and $q_{2}$. The supports of $q_{1}$ and $q_{2}$ are disjoint, and for $i=1,2, q_{i}>0$ only when $\phi>0$.

Observe that $P_{1}$ and $P_{2}$ are mutually absolutely continuous. Set $P_{i}(\cdot)=P_{P_{i}}(\cdot)$, and let $E_{i}$ denote the expectation under $P_{i}$ for $i=1,2$. Using the usual change of measure, we have that

$$
\begin{aligned}
q_{2}(z) d z & =P_{2}(Z \in d z) \\
& =E_{1} I(Z \in d z) \prod_{i=1}^{T} \frac{p_{2}\left(X_{i}\right)}{p_{1}\left(X_{i}\right)} .
\end{aligned}
$$

Now, let $A_{1}$ be such that $Q_{1}\left(A_{1}\right)=1$ and $Q_{2}\left(A_{1}\right)=0$. We can choose such a set since the supports of $q_{1}$ and $q_{2}$ are disjoint. We then arrive at the contradiction

$$
\begin{aligned}
0 & =Q_{2}\left(A_{1}\right) \\
& =\int_{A_{1}} q_{2}(z) d z \\
& =E_{1} I\left(Z \in A_{1}\right) \prod_{i=1}^{T} \frac{p_{2}\left(X_{i}\right)}{p_{1}\left(X_{i}\right)} \\
& =E_{1} \prod_{i=1}^{T} \frac{p_{2}\left(X_{i}\right)}{p_{1}\left(X_{i}\right)} \\
& =1
\end{aligned}
$$

where (4) holds since $Q_{1}\left(A_{1}\right)=P_{1}\left(Z \in A_{1}\right)=1$, and (5) follows since $T$ is a finite-valued stopping time.

One might argue that $\mathcal{P}_{1}$ is unrealistically rich in that it includes probabilities $P$ such that their $Q$-components are mutually singular. This is, in fact, the basis for 
the proof of Theorem 1. In the GSMP context, one might be able to a priori argue that the $Q$-components in question must have a component that is common. This amounts to restricting the set $\mathcal{P}_{0}$ to another subclass $\mathcal{P}_{2}$. Let $\eta$ be a probability measure on $(\mathbb{R}, \mathcal{B})$, and set

$$
\mathcal{P}_{2}=\left\{P \in \mathcal{P}_{0}: P=\lambda \varphi+(1-\lambda) Q, \text { where } Q \text { is equivalent to } \eta\right\} .
$$

Even in the class $\mathcal{P}_{2}$, one cannot create the desired algorithm.

Theorem 2 Suppose that $\lambda \in(0,1)$ and $\varphi$ has a density $\phi$. Then there does not exist a pair $(Z, T)$ as defined above that satisfies property (3) for all $P \in \mathcal{P}_{2}$.

Proof: Consider a sequence $P_{n} \in \mathcal{P}_{2}$ with densities $p_{n}$ such that

$$
p_{n}=\lambda \phi+(1-\lambda) q_{n} .
$$

Select $q_{n}$ so that $q_{n}$ is positive if and only if $\phi$ is positive (and zero otherwise), thereby ensuring that all of the $Q_{n}$ s are equivalent to $\eta=\varphi$. Then for all $B \in \mathcal{B}$,

$$
\begin{aligned}
Q_{n}(B) & =\int_{B} q_{n}(z) d z \\
& =E_{1} I(Z \in B) \prod_{i=1}^{T} \frac{p_{n}\left(X_{i}\right)}{p_{1}\left(X_{i}\right)} \\
& =E_{1} I(Z \in B) \prod_{i=1}^{T} \frac{\lambda \phi\left(X_{i}\right)+(1-\lambda) q_{n}\left(X_{i}\right)}{\lambda \phi\left(X_{i}\right)+(1-\lambda) q_{1}\left(X_{i}\right)} .
\end{aligned}
$$

We further select $Q_{n}$ so that there exists a partition $A_{1}, A_{2}, A_{3} \in \mathcal{B}$ of $\mathbb{R}$ on which $q_{n}(z) \downarrow 0$ as $n \rightarrow \infty$ for all $z \in A_{1}, q_{n}(z) \uparrow q_{\infty}(z)$ as $n \rightarrow \infty$ for all $z \in A_{2}$, and $q_{n}(z)=0$ for all $z \in A_{3}$; see Figure 2. (Note that $A_{3}$ represents the complement of the support of $\varphi$.)

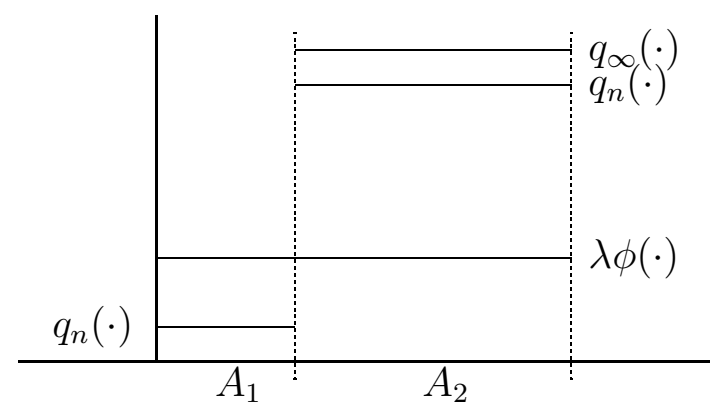

Figure 2: An example of the densities $q_{n}$ in Theorem 2. The sets $A_{1}$ and $A_{2}$ are the left and right intervals respectively. Here $A_{1} \cup A_{2}=[0,1], q_{n}(z)=1 / n$ on $A_{1}$, while $q_{n}(z)=\left(1-\mu\left(A_{1}\right) / n\right) / \mu\left(A_{2}\right)$ on $A_{2}$, where $\mu$ is Lebesgue measure.

Since $q_{n}(z) \downarrow 0$ as $n \rightarrow \infty$ for all $z \in A_{1}$, it follows that $q_{n}(z) \leq q_{1}(z)$ for all $z \in A_{1}$. The dominated convergence theorem then implies that

$$
\begin{aligned}
\lim _{n \rightarrow \infty} Q_{n}\left(A_{1}\right) & =\lim _{n \rightarrow \infty} \int_{A_{1}} q_{n}(z) d z \\
& =\int_{A_{1}} \lim _{n \rightarrow \infty} q_{n}(z) d z \\
& =0 .
\end{aligned}
$$


On the other hand,

$$
\begin{aligned}
\liminf _{n \rightarrow \infty} Q_{n}\left(A_{1}\right) & =\liminf _{n \rightarrow \infty} E_{1} I\left(Z \in A_{1}\right) \prod_{i=1}^{T} \frac{\lambda \phi\left(X_{i}\right)+(1-\lambda) q_{n}\left(X_{i}\right)}{\lambda \phi\left(X_{i}\right)+(1-\lambda) q_{1}\left(X_{i}\right)} \\
& \geq \liminf _{n \rightarrow \infty} E_{1} I\left(Z \in A_{1}\right) \prod_{i=1}^{T} \frac{\lambda \phi\left(X_{i}\right)}{\lambda \phi\left(X_{i}\right)+(1-\lambda) q_{1}\left(X_{i}\right)} \\
& >0
\end{aligned}
$$

where the inequality follows since $U \geq V \geq 0$ implies that $E U \geq E V$. The strict inequality follows since $T$ is a finite-valued stopping time. This is the desired contradiction and the proof is complete.

\section{Discussion}

So, in order to find a pair $(Z, T)$ that works over a class of probabilities, one needs to further restrict $\mathcal{P}_{0}$. Of course, a restriction on $\mathcal{P}_{0}$ amounts to demanding more information about $P \in \mathcal{P}_{0}$, or equivalently, $Q_{P}=(P-\lambda \varphi) /(1-\lambda)$. For example, consider an extreme case. Let $A$ be the support of $\varphi$. Take $\mathcal{P}_{3}$ to be the set of probabilities $P \in \mathcal{P}_{0}$ with the property that the support of $Q_{P}$ does not intersect $A$. Then we can define $T=\inf \left\{n \geq 1: X_{n} \notin A\right\}$ and $Z=X_{T}$.

This extreme case indicates that algorithms of the form discussed in this paper may exist when the class $\mathcal{P}_{0}$ is appropriately restricted, but the negative results in the previous section suggest that any such restrictions will need to be quite severe.

What are the implications of these results for regenerative steady-state simulation of discrete-event systems? Basically, one cannot identify randomized regeneration times of the form described in Section 2 without exploiting some form of information about the $m$-step transition kernel beyond the ability to generate random variates.

Such knowledge is available in nontrivial examples [9]. Unfortunately, in that setting the available knowledge will lead to regenerative cycles with excessive cycle lengths except in examples with relatively few active events.

Of course, these results do not rule out the possibility of identifying nonrandomized regeneration times such as those generated by visits to an atom in a Markov chain. For nontrivial examples of such regeneration times in queueing systems, see $[15,13,14,18]$.

To close the paper, let us return to the "sequence of Bernoulli random variables" example from the introduction. How is this example related to regenerative simulation? Using the notation of Section 3, suppose that $\varphi$ denotes a $\operatorname{Bernoulli}(1 / 2)$ random variable so that $\varphi(\{0\})=\varphi(\{1\})=1 / 2$. Also suppose that $P \in \mathcal{P}_{0}$ is known to correspond to a Bernoulli random variable $X$. We take $\mathcal{P}_{4}=\{P: P(\{1\})=p=1-P(\{0\}), p \in[1 / 4,3 / 4]\}$. Then $P(\cdot) \geq \lambda \varphi(\cdot)$ for all $P \in \mathcal{P}_{4}$ where $\lambda=1 / 2$. For a given $p \in[1 / 4,3 / 4]$, the distribution $Q$ is then easily computed to be $\operatorname{Bernoulli}(2(p-1 / 4))$. Thus, our goal is to generate a $\operatorname{Bernoulli}(2(p-1 / 4))$ random variable from a $\operatorname{Bernoulli}(p)$ random variable, where $p \in[1 / 4,3 / 4]$. The example that opened the paper is an abstraction of this one. The example is further motivated in that it plays a role in attempting to simulate a stationary version of a regenerative process [2].

We are now ready to answer the question posed in the introduction. 
Let $\left(X_{i}: i \geq 1\right)$ be an i.i.d. sequence of $\operatorname{Bernoulli}(p)$ random variables where $p \in[0,1 / 2)$. Let $\left(U_{n}: n \geq 1\right)$ be an i.i.d. sequence of uniformly distributed random variables on $(0,1)$ that is independent of $\left(X_{i}: i \geq 1\right)$. As before let $\mathcal{F}_{n}=\sigma\left(\left(X_{i}, U_{i}\right)\right.$ : $1 \leq i \leq n)$. We are asking whether there exists a stopping time $T$ that is finite a.s., and a random variable $Z$ that is measurable with respect to $\mathcal{F}_{T}$ such that $P(Z=1)=2 p=1-P(Z=0)$.

Proposition 3 Suppose that $p$ can take on any value in the interval $[0,1 / 2)$. Then there does not exist a pair $(Z, T)$ as defined above with the property that $P(Z=$ 1) $=2 p=1-P(Z=0)$.

The proof of Proposition 3 is entirely similar to that of Theorem 2 and so is omitted. Proposition 3 also follows from a result in [11] where the existence of algorithms for generating Bernoulli random variables was explored. We will discuss the main result of [11] in more detail shortly.

We can immediately obtain the following corollary related to the existence or not of an unbiased estimator of $p$. Let $\tau$ be a stopping time with respect to the filtration $\left(\mathcal{F}_{n}: n \geq 1\right)$ that is finite a.s., and $\zeta$ be measurable with respect to $\mathcal{F}_{\tau}$. Here $\zeta$ represents an estimator of $p$ and $\tau$ the "time" required to compute it.

Corollary 4 Suppose that $p$ can take on any value in the interval $[0,1 / 2)$. There does not exist a pair $(\zeta, \tau)$ such that $\zeta$ is an unbiased estimator of $p$ with $\zeta \in[0,1 / 2)$ a.s.

Proof: Suppose that the pair $(\zeta, \tau)$ existed. Then compute the random variable $Z=I(U<2 \zeta)$ where $U=U_{\tau+1}$. Then $Z$ has a Bernoulli distribution and

$$
P(Z=1)=E P(U<2 \zeta \mid \zeta)=E(2 \zeta)=2 p .
$$

Thus the pair $(Z, \tau+1)$ contradicts Proposition 3 .

In fact, the existence of an unbiased estimator of $p$ that lies in the interval $[0,1 / 2)$ almost surely is equivalent to the existence of a pair $(Z, T)$ as in Proposition 3. Suppose that $(Z, T)$ exists, and set $\zeta=0.5 Z$. Then $\zeta$ is an unbiased estimator of $p$ and lies in the interval [0,1/2). A similar observation appears as Remark 3.2 in [2]. Roughly speaking, the result in [2] shows that the existence of an unbiased estimator of the stationary distribution $\pi$ of a finite state space irreducible Markov chain is equivalent to the existence of a method for obtaining "perfect samples" from $\pi$.

Remark 3 There are a myriad of unbiased estimators of $p$, but Corollary 4 shows that none exist that both lie in the interval $[0,1 / 2)$ a.s. and can be computed in finite time.

Remark 4 While one cannot generate a Bernoulli random variable with probability of success exactly equal to $2 p$, one can certainly get arbitrarily close. Let $\tau_{n}=n$, and define

$$
\zeta_{n}=\min \left(\frac{1}{n} \sum_{i=1}^{n} X_{i}, \frac{1-n^{-1}}{2}\right) .
$$

Then construct $Z_{n}$ from $\zeta_{n}$ as in the proof of Proposition 3. Then $P\left(Z_{n}=1\right)=$ $E \zeta_{n} \rightarrow p$ as $n \rightarrow \infty$. 
Based on the arguments presented here, we can say nothing about the existence or nonexistence of an algorithm for generating Bernoulli $(2 p)$ random variables where $p$ can take on any value in the interval $[0,1 / 2-\epsilon)$ for some $\epsilon \in(0,1 / 2)$. However, using an elegant argument, [11] establishes constructively that such an algorithm does indeed exist!

In fact, [11] gives necessary and sufficient conditions on a function $f$ (including its domain) so that one can generate $\operatorname{Bernoulli}(f(p))$ random variables based only on the ability to generate $\operatorname{Bernoulli}(p)$ random variables. Their result may be stated as follows.

Let the filtration $\mathcal{F}=\left(\mathcal{F}_{n}: n \geq 1\right)$ be defined as above.

Theorem 5 (Keane and O'Brien) Let $A \subseteq[0,1]$ and $f: A \rightarrow[0,1]$. Then a pair $(Z, T)$ exists where $T$ is a finite-valued stopping time with respect to $\mathcal{F}, Z$ is measurable with respect to $\mathcal{F}_{T}$ and $Z$ is Bernoulli $(f(p))$ if, and only if,

(i) $f$ is continuous on $A$, and

(ii) either $f$ is constant on $A$, or there exists an integer $n$ such that

$$
\min \{f(p), 1-f(p)\} \geq \min \left\{p^{n},(1-p)^{n}\right\}
$$

for all $p \in A$.

To see why Theorem 5 does not contradict Proposition 3 above, observe that $f(p)=2 p$ defined on the interval $A=[0,1 / 2)$ does not satisfy condition (ii) of Theorem 5. However, $f(p)=2 p$ defined on the interval $A=[0,1 / 2-\epsilon)$ does satisfy the conditions of Theorem 5 .

This result establishes the existence of algorithms of the form discussed in this paper in the very special case where $P, \varphi$ and $Q$ have Bernoulli distributions. However, the delicacy of the conditions and argument in [11], together with the results in Section 3 for more general distributions, suggest that a practical algorithm of the form described here for detecting regenerations in general discrete-event systems does not exist.

\section{Acknowledgments}

We would like to thank Evsey Morozov and Irina Aminova for sharing working versions of their paper [14] with us. The work of the first author was supported by National Science Foundation Grant DMI-0085165. The work of the second author was supported by National Science Foundation Grant DMS-9704732-001.

\section{References}

[1] S. Asmussen. Applied Probability and Queues. Wiley, Chichester, 1987.

[2] S. Asmussen, P. W. Glynn, and H. Thorisson. Stationarity detection in the initial transient problem. ACM Transactions on Modeling and Computer Simulation, 2(2):130-157, 1992.

[3] K. B. Athreya and P. Ney. A new approach to the limit theory of recurrent Markov chains. Transactions of the American Mathematical Society, 245:493501, 1978. 
[4] P. Billingsley. Probability and Measure. Wiley, New York, 2nd edition, 1986.

[5] H. Damerdji and P. W. Glynn. Limit theory for performance modeling of future event set algorithms. Management Science, 44(12):1709-1718, 1998.

[6] P. W. Glynn. Some topics in regenerative steady-state simulation. Acta Applicandae Mathematicae, 34:225-236, 1994.

[7] P. W. Glynn and P. L'Ecuyer. Likelihood ratio gradient estimation for stochastic recursions. Advances in Applied Probability, 27:1019-1053, 1993.

[8] P. J. Haas. On simulation output analysis for generalized semi-Markov processes. Communications in Statistics: Stochastic Models, 15:53-80, 1999.

[9] S. G. Henderson and P. W. Glynn. Can the regenerative method be applied to discrete-event simulation? In P. A. Farrington, H. Black Nembhard, D. T. Sturrock, and G. W. Evans, editors, Proceedings of the 1999 Winter Simulation Conference, pages 367-373, Piscataway NJ, 1999. IEEE.

[10] S. G. Henderson and P. W. Glynn. Regenerative steady-state simulation of discrete event systems. ACM Transactions on Modeling and Computer Simulation, To appear, 2001.

[11] M. S. Keane and G. L. O'Brien. A Bernoulli factory. ACM Transactions on Modeling and Computer Simulation, 4(2):213-219, 1994.

[12] S. P. Meyn and R. L. Tweedie. Markov Chains and Stochastic Stability. Springer-Verlag, London, 1993.

[13] E. Morozov. Weak regenerative structure of open Jackson queueing networks. Journal of Mathematical Sciences, 91:2956-2961, 1998.

[14] E. Morozov and I. Aminova. On steady-state simulation of some weak regenerative networks. Preprint, 2001.

[15] E. Morozov and S. Sigovtsev. Simulation of queueing processes based on weak regeneration. Journal of Mathematical Sciences, 89:1517-1523, 1998.

[16] E. Nummelin. A splitting technique for Harris recurrent Markov chains. $Z$. Wahrsch. Verw. Gebiete, 43(4):309-318, 1978.

[17] G. S. Shedler. Regenerative Stochastic Simulation. Academic Press, Boston, 1993.

[18] K. Sigman. Regeneration in tandem queues with multiserver stations. Journal of Applied Probability, 25:391-403, 1988.

[19] K. Sigman. One-dependent regenerative processes and queues in continuous time. Mathematics of Operations Research, 15(1):175-189, 1990. 